\title{
Severe urinary retention secondary to intrathecal morphine pain pump: a case report
}

\author{
Eyitemi Fregene $^{1}$ and Peter Lotze ${ }^{2}$ \\ ${ }^{1}$ University of Houston \\ ${ }^{2}$ The Woman's Hospital of Texas
}

November 15, 2021

\begin{abstract}
An elderly female with multiple comorbidities presented with acute onset of severe urinary retention immediately following placement of an intrathecal morphine pain pump. This case highlights the need to closely monitor chronic pain patients with complex medical histories who may be uniquely predisposed to opioid-mediated severe urinary retention.
\end{abstract}

Title: Severe urinary retention secondary to intrathecal morphine pain pump: a case report

Keywords : neurogenic bladder, intrathecal morphine, urinary retention, pain pump, nonmalignant pain

Authors : E. Fregene, MD, MPH, P. Lotze, MD

Corresponding author contact info :

Eyitemi Fregene, MD, MPH

Email: Eyitemi.Fregene@hcahealthcare.com

Statement on journal ethics and integrity policies : Wiley publishing ethics and integrity guidelines have been reviewed. This manuscript is complaint with Wiley publishing ethics and integrity guidelines.

Data availability statement : Wiley's four policies on data sharing have been reviewed. Data referenced in the case below is available upon request.

Funding statement : No funding was provided for this case report.

Conflict of interest statement : There are no conflicts of interest to disclose.

Institutional Review Board Statement : This research activity was determined to be exempt or excluded from Institutional Review Board (IRB) oversight in accordance with current regulations and institutional policy. Our internal reference number for this determination is 2021-727. All research materials provided herein, in whole or in part, are subject to copyright protection by HCA Healthcare and/or one of its subsidiaries. All rights reserved.

Patient consent : Written consent to write and publish this case report were obtained from the patient who is the subject of the case report.

\section{Abstract :}

An elderly female with multiple comorbidities presented with acute onset of severe urinary retention immediately following placement of an intrathecal morphine pain pump. This case highlights the need to 
closely monitor chronic pain patients with complex medical histories who may be uniquely predisposed to opioid-mediated severe urinary retention.

\section{Introduction}

Intrathecal pain pumps (IPP) are sometimes prescribed by pain medicine specialists for treatment of chronic nonmalignant pain. Urinary retention is a well-recognized side effect of opioids administration via IPP for chronic nonmalignant pain. Mild urinary retention, not requiring intervention, following intrathecal morphine administration has an estimated incidence of approximately $42 \%$ in nonmalignant pain patients ${ }^{1-3}$. Severe urinary retention in this context is less common. A large retrospective study on intrathecal morphine in 39 nonmalignant chronic pain patient describes a single case of severe urinary retention $(2.6 \%)$ as a complication of intrathecal analgesia requiring system removal ${ }^{4}$.

We were able to identify only one case report in the literature which focused on management strategies for resolving urinary retention secondary to IPP placement ${ }^{5}$. In this case report, the urinary retention was resolved 4 days after reducing the morphine infusion rate by $75 \%$ from $2000 \mathrm{mcg} / 24$ hours to $500 \mathrm{mcg} /$ 24 hours $^{5}$. We present our experience with a case of a neurogenic bladder which presented as severe urinary retention immediately following IPP placement for chronic peripheral neuropathy in an elderly female with multiple comorbidities. Dose reduction did not remedy the urinary retention. The urinary retention only improved after changing the type of narcotic infused.

\section{Case history:}

A 70-year-old female underwent placement of a morphine Prometra IPP (Flowonix, Mt. Olive, NJ) for chronic peripheral neuropathy. The IPP was implanted by her pain management specialist. The patient's history was significant for chronic pain syndrome, peripheral vascular disease, prior cerebrovascular accident with no residual deficits, hypertensive disorder, 10-year history of type II diabetes, and spastic pelvic floor muscles. At the time of symptom onset, the patient had no history of urinary retention, voiding dysfunction, pelvic organ prolapse, or urinary incontinence.

The patient initially presented to urogynecology 4 days after morphine IPP placement complaining that she was "unable to void without assistance." Urinalysis and urine culture were negative for infection. Voided volumes were less than $100 \mathrm{cc}$. Her postvoid residual volumes (PVRs) were approximately $600 \mathrm{cc}$, consistent with acute urinary retention.

\section{Differential diagnosis, investigations and treatment}

The differential for the acute urinary retention included pelvic floor muscle spasticity, narcotic exposure, and/or vasculopathy secondary to multiple comorbidities. In our patient's case, her narcotic exposure played the primary role in her retention complaint.

The patient, a former nurse, began intermittent self-catheterization (ISC) upon its onset and began a trial of tamsulosin. Experiencing no benefit with the tamsulosin one week later, the patient declined to continue ISC and stopped the medication. An indwelling urinary catheter was placed. Nitrofurantoin was prescribed for infection prophylaxis while the indwelling catheter was in place.

Multiple voiding trials were performed for up to three weeks following symptom onset. Further diagnostic testing was performed. Cystoscopy showed diffuse inflammation and moderate trabeculations. Urodynamics demonstrated decreased bladder compliance during filling with a decreased bladder capacity. Voiding pressure studies revealed minimal detrusor contraction with a valsalva effort and an intermittent voiding pattern with minimal output. EMG demonstrated no abnormalities during testing.

To determine if the morphine IPP was related to the patient's urinary retention, the pain management specialist decreased the basal rate of the IPP from morphine sulfate $100 \mathrm{mcg}$ daily to $90 \mathrm{mcg}$ daily. However, the basal rate was increased back to the initial rate within 5 days due to patient complaints of burning in her legs related to peripheral neuropathy. 


\section{Outcome and follow-up}

After completion of the urogynecology workup which failed to demonstrate any evidence of other etiology to account for retention complaints, recommendations were made to turn off the pain pump. Ultimately, the IPP was disabled and a voiding trial was performed 48 hours later. The patient was then able to void spontaneously with PVRs less than $150 \mathrm{cc}$. During this time, the patient took one tablet of acetaminophen $300 \mathrm{mg}$-codeine $30 \mathrm{mg}$ orally every 6 hours. Morphine sulfate was identified as the cause of urinary retention. She was transitioned to intrathecal fentanyl for pain management. She repeatedly demonstrated both objective and subjective evidence of adequate voiding with post-void residuals of approximately $150 \mathrm{cc}$.

\section{Discussion}

Our patient was likely predisposed to experience severe urinary retention given her complex medical history. The following basic physiology is recognized as the scientific basis of severe urinary retention in the setting of intrathecal opioid administration: the opioids receptors involved in the urodynamic effects are $\mu$ and $\delta$. Intrathecal opioids acting on opioid receptors in the spinal cord decrease the parasympathetic firing in the sacral region and decrease the afferent inputs from the bladder to the spinal cord which results in an inhibitory modulating effect on the release of acetylcholine that causes detrusor contraction ${ }^{6}$. Some speculate that there is a dose-dependent mechanism by which intrathecal opioids cause side effects like urinary retention ${ }^{1}$. Other sources describe a primary dose-independent activation of opioid receptors ${ }^{7}$. A randomized, doubleblind study by Raffaeli et al. concluded that side effects of intrathecal opioid like urinary retention can be considered as a patient-dependent effect of the drug, suggesting the presence of a primary dose-independent excitatory component that might be related to the theory of the bimodal activation of opioid receptors. Our case supports this latter theory because dose reduction did not improve the patient's severe urinary retention.

The improvement of the patient's urinary retention was achieved by changing the type of narcotic. This demonstrates how different narcotics may vary in how they affect the $\mu$ and $\delta$ receptors resulting in unique, narcotic-specific outcomes with respect to pain management and risk of voiding dysfunction.

\section{Conclusion :}

Morphine sulfate was successfully identified as the cause of the new-onset urinary retention after turning off the morphine IPP. This patient has a complex medical history including stroke with no residual deficits, diabetic neuropathy, and peripheral vascular disease that may have contributed to her susceptibility to severe urinary retention. The patient's initial complaint of difficulty urinating and her urodynamic testing findings may reflect the influence of intrathecal opioid administration as opioids are known to decrease bladder function by suppressing detrusor contractility and decreasing the sensation of urge ${ }^{8}$. Of note, different types of opioids may uniquely affect bladder storage and emptying demonstrated during urodynamics. For instance, drugs like fentanyl are more lipophilic than morphine and thus undergo greater systemic uptake. As a result, there is less rostral spread in the central nervous system and less influence on storage and voiding as demonstrated by multichannel urodynamics. Published research also supports replacing morphine with an alternative narcotic such as sufentanil in a regional anesthetic regimen to mitigate the risk of postoperative

urinary retention ${ }^{9}$. The data indicate that recovery to normal lower urinary tract function is significantly faster after intrathecal sufentanil than morphine ${ }^{6,9}$.

This case report highlights that chronic pain patients with multiple comorbidities affecting the nervous system may be uniquely predisposed to severe urinary retention with IPP use. Intrathecal morphine may be more likely to precipitate urinary retention in such a patient. We believe that patients with a morphine IPP in retention should undergo a trial of an alternative narcotic as a primary management strategy to resolve the urinary retention and maintain the benefit of the IPP.

\section{References}

1. Ruan X. Drug-related side effects of long-term intrathecal morphine therapy. Pain Physician. 2007 Mar;10(2):357-66. PMID: 17387357. 
2. Turner JA, Sears JM, Loeser JD. Programmable intrathecal opioid delivery systems for chronic noncancer pain: a systematic review of effectiveness and complications. Clin J Pain. 2007 Feb;23(2):180-95. doi: 10.1097/01.ajp.0000210955.93878.44. PMID: 17237668.

3. Winkelmüller M, Winkelmüller W. Long-term effects of continuous intrathecal opioid treatment in chronic pain of nonmalignant etiology. J Neurosurg. 1996 Sep;85(3):458-67. doi: 10.3171/jns.1996.85.3.0458. PMID: 8751633.

4. Gay ML. Spinal morphine in nonmalignant chronic pain: a retrospective study in 39 patients. Neuromodulation. 2002 Jul;5(3):150-9. Doi: 10.1046/j.1525-1403.2002.02025.x. PMID: 22150812.

5. Uppal GS, Haider TT, Dwyer A, Uppal JA. Reversible urinary retention secondary to excessive morphine delivered by an intrathecal morphine pump. Spine (Phila Pa 1976). 1994 Mar 15;19(6):719-20. doi: 10.1097/00007632-199403001-00013. PMID: 8009339.

6. Baldini G, Bagry H, Aprikian A, Carli F, Warner D, Warner M; Postoperative Urinary Retention: Anesthetic and Perioperative Considerations. Anesthesiology 2009; 110:1139-1157 doi https://doi.org/10.1097/ALN.0b013e31819f7aea.

7. Raffaeli W, Marconi G, Fanelli G, Taddei S, Borghi GB, Casati A. Opioid-related side-effects after intrathecal morphine: a prospective, randomized, double-blind dose-response study. Eur J Anaesthesiol. 2006 Jul;23(7):605-10. doi: 10.1017/S026502150600038X. Epub 2006 Mar 1. PMID: 16507190.

8. Kuipers PW, Kamphuis ET, van Venrooij GE, van Roy JP, Ionescu TI, Knape JT, Kalkman CJ: Intrathecal opioids and lower urinary tract function: A urodynamic evaluation. Anesthesiology 2004; 100:1497-503.

9. Jackson, J., Davies, P., Leggett, N., Nugawela, M.D., Scott, L.J., Leach, V., Richards, A., Blacker, A., Abrams, P., Sharma, J., Donovan, J., \& Whiting, P. (2019, February). Systematic review of interventions for the prevention and treatment of postoperative urinary retention: Prevention and treatment of postoperative urinary retention. BJS Open , 3 (1), 11-23.https://doi.org/10.1002/bjs5.50114.

Disclaimer: This research was supported (in whole or in part) by HCA Healthcare and/or an HCA Healthcare affiliated entity. The views expressed in this publication represent those of the author(s) and do not necessarily represent the official views of HCA Healthcare or any of its affiliated entities. 